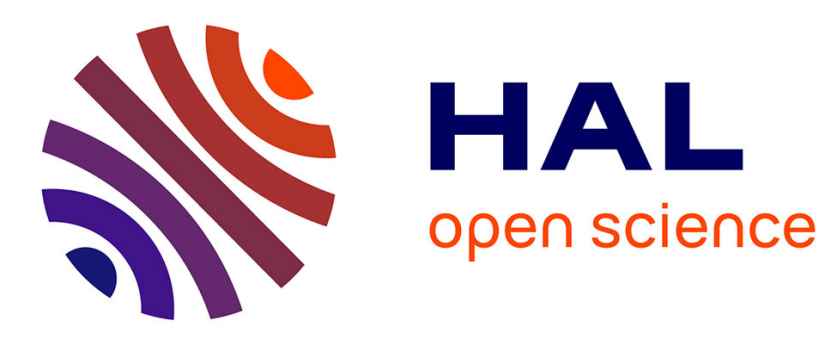

\title{
Predictive scheme for observer-based control of LTI systems with unknown disturbances
}

Vincent Léchappé, Emmanuel Moulay, Franck Plestan, Alain Glumineau, Abdelhamid Chriette

\section{- To cite this version:}

Vincent Léchappé, Emmanuel Moulay, Franck Plestan, Alain Glumineau, Abdelhamid Chriette. Predictive scheme for observer-based control of LTI systems with unknown disturbances. European Control Conference, Jul 2015, Linz, Austria. 10.1109/ECC.2015.7330841 . hal-01179420

\section{HAL Id: hal-01179420 \\ https://hal.science/hal-01179420}

Submitted on 26 Jan 2016

HAL is a multi-disciplinary open access archive for the deposit and dissemination of scientific research documents, whether they are published or not. The documents may come from teaching and research institutions in France or abroad, or from public or private research centers.
L'archive ouverte pluridisciplinaire HAL, est destinée au dépôt et à la diffusion de documents scientifiques de niveau recherche, publiés ou non, émanant des établissements d'enseignement et de recherche français ou étrangers, des laboratoires publics ou privés. 


\title{
Predictive scheme for observer-based control of LTI systems with unknown disturbances
}

\author{
V. Léchappé, E. Moulay, F. Plestan, A. Glumineau and A. Chriette
}

\begin{abstract}
Index Terms - Input delay, unknown disturbance, reduction method, predictive control, observation.

Abstract-In this work, it is shown that the results introduced in [1], that hold for full state measurement, can be extended to partial state measurement. In particular, it is proven that the combination of an observer with the new predictive scheme of [1] leads to a better disturbance attenuation than using the same observer with the standard predictive scheme. Finally, some simulations illustrate the results for constant and timevarying disturbances.
\end{abstract}

\section{INTRODUCTION}

One of the first work on the control of input delay systems is the well-known Smith predictor [2]. It is a frequency domain approach for SISO and open-loop stable systems. In the 80's, the Finite Spectrum Assignment (FSA) [3], [4] and the model reduction, also called Artstein reduction [5], have extended Smith's work to MIMO, open-loop unstable systems. All these techniques lead to state feedback controllers because they use the prediction and the full state has to be known to compute the prediction. Furthermore, these reference articles do not deal with disturbance attenuation. A complete analysis of predictive control can be found in [6].

In spite of numerous works on Time Delay Systems (TDS), very few articles deal with disturbance attenuation in presence of delay in the input even if there is a real interest from a practical point of view. Indeed, it is really a complex challenge even for linear systems. Some robust control methods have been extended to input delay systems. First, sliding mode control, known for its robustness in the delay-free case, has been adapted to input delay systems by using "surfaces" [7], [8] or a standard surface [9], [10]. The problem of sliding mode with relay systems is the unavoidable apparition of oscillations [11]. $H_{\infty}$ control has also been studied for input delay systems and a review is proposed in [12]. A complete analysis of this topic is also provided by [13]. All these works consider that the whole state is available for measurement.

On the contrary, many works tackle the observer-based control problem but in the disturbance free case. The problem of state reconstruction for system with delayed input has been addressed first in [14] and [15]. Then, their works have been extended to observer-based control. In [16], a predictive feedback control from the reconstructed states is designed.

\footnotetext{
Vincent Léchappé, Franck Plestan, Alain Glumineau and Abdelhamid Chriette are with LUNAM Université, Ecole Centrale de Nantes, IRCCyN UMR CNRS 6597, Nantes, France. Firstname. Namedirccyn.ec-nantes.fr.

Emmanuel Moulay is with Xlim, UMR CNRS 7252, Université de Poitiers emmanuel.moulay@univ-poitiers.fr
}

The problem of output feedback for nonlinear systems is addressed in [17] but the designed controller does not involve any prediction. In [18], the controller is a Truncated Predictor Feedback (TPF) introduced in [19].

To the authors knowledge, there exist only two works combining output predictive feedback and perturbation attenuation. In the discrete framework, [20] uses a disturbance observer and a linear predictive feedback. In [21], a geometric approach is used to show the existence of an output predictive feedback that minimizes the effect of the disturbance on the system. However, they use the standard prediction so the disturbance attenuation can be improved by using the new predictive scheme proposed in [1].

This work extends the results of [1] to the systems with partial state measurement. The combination of an observer with the new predictive scheme allows the design of controllers that performs a better disturbance attenuation than the standard reduction method. This extension is particularly interesting from a practical point of view.

The paper is organized as follows. The problem formulation is given in Subsection II-A and the predictive schemes with full state feedback is recalled in II-B. The predictive schemes for partial state measurement is introduced at the end of Section II. Section III provides a comparison between the standard and the new predictive schemes with partial state measurement. Theoretical results are illustrated by simulations in Section IV. Finally, some conclusions are drawn in Section V.

\section{Problem STATEMENT AND PREDictive APPROACHES}

\section{A. Problem Statement}

The considered systems are LTI systems with a delay $h$ acting on the control input $u$ and an additive disturbance $d$

$$
\left\{\begin{array}{l}
\dot{x}(t)=A x(t)+B u(t-h)+d(t) \\
y(t)=C x(t) \\
u(t)=u_{0}(t) \quad \text { for all } t \in[-h, 0[ \\
x(0)=x_{0}
\end{array}\right.
$$

with $x(t) \in \mathbb{R}^{n}, u(t) \in \mathbb{R}^{m}, d(t) \in \mathbb{R}^{n}, A \in \mathbb{R}^{n \times n}, B \in$ $\mathbb{R}^{n \times m}$ and $C \in \mathbb{R}^{p \times n}$.

Assumption 1: $A, B$ and $C$ are constant and known, the pair $(A, B)$ is controllable, the pair $(C, A)$ is observable.

\section{Assumption 2: $h>0$ is constant and known.}

Let $I \subseteq \mathbb{R}$ be an unbounded interval and $S \subseteq \mathbb{R}^{m}$ be a set. The space of locally integrable functions $u($.$) defined on I$ and taking values into $S$ is denoted by $L_{l o c}^{1}(I, S)$. 
Assumption 3: $u$ is a locally integrable function: $u \in$ $L_{\text {loc }}^{1}\left(\left[-h,+\infty\left[, \mathbb{R}^{m}\right)\right.\right.$.

Assumption 4: $d: \mathbb{R}_{\geq 0} \rightarrow \mathbb{R}^{n}$ is an unknown function such that for all $t \geq 0$,

$$
\|d(t)\| \leq d_{\max }<+\infty
$$

and for all $t \geq h$,

$$
\|d(t)-d(t-h)\| \leq h D_{\max }<+\infty .
$$

The inequality (3) is implied by (2) choosing $D_{\max }=\frac{2 d_{\max }}{h}$ but more accurate upper bound may exist.

\section{B. Predictive schemes with full state knowledge}

In this subsection, the new predictive scheme designed in [1] is recalled. This scheme is based on the definition of a new prediction $X_{\hat{p}}$ :

Definition 1: Let us define:

$$
X_{\hat{p}}(t)=x_{\hat{p}}(t)+x(t)-x_{\hat{p}}(t-h)
$$

for all $t \geq h$, with $x_{\hat{p}}$ given by

$$
x_{\hat{p}}(t)=e^{A h} x(t)+\int_{t-h}^{t} e^{A(t-s)} B u(s) \mathrm{d} s .
$$

The integral term in (5) and all the integral terms mentioned in the sequel are well defined thanks to Assumptions 3 and 4.

The term $x_{\hat{p}}$ is the standard prediction that is used in almost all papers about predictive control of input delay systems [5],[4], [2]. Basicaly, $X_{\hat{p}}(t)$ is equal to the standard prediction $x_{\hat{p}}(t)$ corrected by $x(t)-x_{\hat{p}}(t-h)$. This latter term represents the prediction error at instant $t-h$ due to the unknown perturbation $d$ :

$$
x(t)-x_{\hat{p}}(t-h)=\int_{t-h}^{t} e^{A(t-s)} d(s) \mathrm{d} s
$$

Note that the computation of $X_{\hat{p}}$ does not require any knowledge of the perturbation but it requires the full knowledge of $x$.

The reduction method consists in rewritting system (1) in terms of predictions ${ }^{1}$. For the standard prediction $x_{\hat{p}}$, the transformation leads to the delay free system:

$$
\dot{x}_{\hat{p}}(t)=A x_{\hat{p}}(t)+B u(t)+e^{A h} d(t) .
$$

For the new prediction $X_{\hat{p}}$, it yields to

$$
\dot{X}_{\hat{p}}(t)=A X_{\hat{p}}(t)+B u(t)+d(t)+e^{A h}[d(t)-d(t-h)] .
$$

Then, the objective consists in designing the control law $u$ on delay free systems (6) or (7). In the rest of the paper,

- designing a controller based on the standard predictive sheme will refer to the design of a control law on (6) so the controller will read as $u=f\left(x_{\hat{p}}\right)$;

\footnotetext{
${ }^{1}$ In the Artstein reduction method [5], the system is turned into a delay free system using the transformation $Z(t)=e^{-A h} x_{\hat{p}}(t)$.
}

- designing a controller based on the new predictive scheme will refer to the design of a control law on (7) so the controller will read as $u=f\left(X_{\hat{p}}\right)$.

It has been shown in [1] that a controller of the form $u(t)=f\left(X_{\hat{p}}\right)$ (with $f$ is a Lipschitz continuous function) leads to a better disturbance attenuation than a controller of the form $u(t)=f\left(x_{\hat{p}}\right)$ for a wide class of perturbations. In next subsection, previous considerations are extended to the partial state measurement case.

\section{Predictive schemes with partial state knowledge}

The computation of $X_{\hat{p}}$ involves the full knowledge of state $x$ in (4). However, when only a part of the state is available, one can design an observer to first recontruct the state and then compute the prediction in a similar way to (4). Consider the Luenberger observer for (1):

$$
\dot{\hat{x}}(t)=A \hat{x}(t)+B u(t-h)+L(y(t)-C \hat{x}(t)) .
$$

Note that (8) can be implemented because $h$ is known. The coefficient $L$ has to be properly chosen such that the following error dynamics is practically stable ${ }^{2}$

$$
\dot{e}(t)=A e(t)-L C e(t)+d(t)
$$

with $e(t)=x(t)-\hat{x}(t)$. In particular, $L$ can be chosen such that the convergence error of the nominal system, with $d(t)=0$, is exponentially stable. Observability Assumption 1 guarantees the existence of such a gain.

From Lemma 9.2 in [23] and Assumption 4, one can state that there exists $T, \Gamma>0$ such that for all $t>T$

$$
\|e(t)\| \leq \Gamma d_{\max }
$$

and

$$
\|e(t)-e(t-h)\| \leq h \Gamma D_{\max } .
$$

From the estimated state $\hat{x}$, we can compute $\hat{X}_{\hat{p}}$, the "reconstructed new prediction":

Definition 2: Let us define:

$$
\hat{X}_{\hat{p}}(t)=\hat{x}_{\hat{p}}(t)+\hat{x}(t)-\hat{x}_{\hat{p}}(t-h)
$$

for all $t \geq h$, with $\hat{x}_{\hat{p}}$ given by

$$
\hat{x}_{\hat{p}}(t)=e^{A h} \hat{x}(t)+\int_{t-h}^{t} e^{A(t-s)} B u(s) \mathrm{d} s .
$$

Like in subsection II-B, the dynamics of (1) can be rewritten in the coordinate of the reconsructed predictions. The reduction in $\hat{x}_{\hat{p}}$ leads to the system :

$$
\dot{\hat{x}}_{\hat{p}}(t)=A \hat{x}_{\hat{p}}(t)+B u(t)+\underbrace{e^{A h} L C e(t)}_{d_{\hat{x}}(t)} .
$$

\footnotetext{
${ }^{2}$ Practical stability means that the observation error $e$ converges to a ball of radius $r>0$ around the origin [22].
} 
As for the transformation with $\hat{X}_{\hat{p}}$, one obtains

$$
\dot{\hat{X}}_{\hat{p}}(t)=A \hat{X}_{\hat{p}}(t)+B u(t)+\underbrace{L C e(t)+e^{A h} L C[e(t)-e(t-h)]}_{d_{\hat{X}}(t)} .
$$

The objective of the next section is to show that the results of [1] still holds when the state is partially known. In other words, it will be proved that designing a controller on (14) $\left(u(t)=f\left(\hat{x}_{\hat{p}}\right)\right)$ leads to better disturbance attenuation than designing a controller on (15) $\left(u=f\left(\hat{X}_{\hat{p}}\right)\right)$. Similarly, to the full state knowledge case,

- designing a controller based on the standard reconstructed predictive sheme will refer to the design of a control law on (14) so the controller will read as $u(t)=f\left(\hat{x}_{\hat{p}}\right)$

- designing a controller based on the new reconstructed predictive scheme will refer to the design of a control law on (15) so the controller will read as $u=f\left(\hat{X}_{\hat{p}}\right)$.

\section{COMPARISON BETWEEN PREDICTIVE SCHEMES WITH PARTIAL MEASUREMENT}

For time varying perturbations, i.e when $D_{\max }>0$, asymptotic stability cannot be achieved, only stability within a ball around the origin is possible. The objective of this subsection is to study the influence of the prediction scheme on the error bound.

Let $f$ be a Lipschitz continuous function and assume the control

$$
u(t)=f\left(\hat{x}_{\hat{p}}(t)\right)
$$

is such that the origin of the closed-loop system (14)-(16) with $d_{\hat{x}}=0$ is a globally exponentially stable equilibrium point. The function $f: \mathbb{R}^{n} \rightarrow \mathbb{R}^{m}$ is locally Lipschitz and $\hat{x}_{\hat{p}}$ is continuous so Assumption 3 holds. Besides, Theorem 4.14 in [23] guarantees the existence of a Lyapunov function $V\left(\hat{x}_{\hat{p}}\right)$ that satisfies

$$
\begin{aligned}
& c_{1}\left\|\hat{x}_{\hat{p}}\right\| \leq V\left(\hat{x}_{\hat{p}}\right) \leq c_{2}\left\|\hat{x}_{\hat{p}}\right\| \\
& \dot{V}\left(\hat{x}_{\hat{p}}\right) \leq-c_{3}\left\|\hat{x}_{\hat{p}}\right\| \\
& \left\|\frac{d V}{d \hat{x}_{\hat{p}}}\right\| \leq c_{4}\left\|\hat{x}_{\hat{p}}\right\|
\end{aligned}
$$

with $c_{1}, c_{2}, c_{3}$ and $c_{4}$ positive constants. In addition, the perturbation of system (14) is bounded and the following maximization holds

$$
\left\|d_{\hat{x}}(t)\right\| \leq \Gamma\left\|e^{A h}\right\|\|L C\| d_{\max }, \quad \forall t \geq T .
$$

Therefore, the assumptions of Lemma 9.4 in [23] are fulfilled (equations (17) and (18)) so one deduces that for all $t \geq T$

$$
\left\|\hat{x}_{\hat{p}}(t)\right\| \leq \beta e^{-\alpha(t-T)}+\gamma \Gamma\|L C\|\left\|e^{A h}\right\| d_{\max }
$$

with $\alpha, \beta$ and $\gamma$ positive constants that depends on $c_{1}, c_{2}$, $c_{3}$ and $c_{4}$.

Since (6) and (7) have the same form when there is no perturbation, i.e $d_{\hat{x}}(t)=0$ and $d_{\hat{X}}(t)=0$, the controller

$$
u(t)=f\left(\hat{X}_{\hat{p}}(t)\right)
$$

guarantees that $\hat{X}_{\hat{p}}=0$ is a globally exponentially stable equilibrium point of the closed-loop system (15)-(20) with $d_{\hat{X}}(t)=0$ for all $t>0$. Similarly to $u(t)=f\left(\hat{x}_{\hat{p}}(t)\right)$, Assumption 3 is verified for $u(t)=f\left(\hat{X}_{\hat{p}}(t)\right)$. Besides, inequalities (17) still holds for $\hat{X}_{\hat{p}}$ :

$$
\begin{aligned}
& c_{1}\left\|\hat{X}_{\hat{p}}\right\| \leq V\left(\hat{X}_{\hat{p}}\right) \leq c_{2}\left\|\hat{X}_{\hat{p}}\right\| \\
& \dot{V}\left(\hat{X}_{\hat{p}}\right) \leq-c_{3}\left\|\hat{X}_{\hat{p}}\right\| \\
& \left\|\frac{d V}{d \hat{X}_{\hat{p}}}\right\| \leq c_{4}\left\|\hat{X}_{\hat{p}}\right\| .
\end{aligned}
$$

From Assumption 4, the inequality

$$
\left\|d_{\hat{X}}(t)\right\| \leq \Gamma|| L C \|\left[d_{\max }+h\left\|e^{A h}\right\| D_{\max }\right],
$$

is verified for all $t \geq T$. Relations (21) and (22) comply with the assumptions of Lemma 9.4 in [23] so the following inequality is obtained

$$
\left\|\hat{X}_{\hat{p}}(t)\right\| \leq \beta e^{-\alpha(t-T)}+\gamma \Gamma\|L C\|\left[d_{\max }+h\left\|e^{A h}\right\| D_{\max }\right]
$$

for all $t \geq T$. The constants $\alpha, \beta$ and $\gamma$ are the same as in (19) because they only depend on the form of the undisturbed system. As it has been mentioned before, systems (14) and (15) have the same representation $\dot{\chi}=A \chi+B u(t)$ when there is no observation error. Since $x$ is the solution of (1),

$$
x(t+h)=e^{A h} x(t)+\int_{t-h}^{t} e^{A(t-s)}[B u(s)+d(s+h)] \mathrm{d} s
$$

and one has

$$
\hat{x}_{\hat{p}}(t)-x(t+h)=-e^{A h} e(t)-\int_{t-h}^{t} e^{A(t-s)} d(s+h) \mathrm{d} s .
$$

After evaluating above expression in $t-h$, it follows that

$$
\|x(t)\| \leq\left\|\hat{x}_{\hat{p}}(t-h)\right\|+\eta d_{\max }+\left\|e^{A h}\right\|\|e(t-h)\|
$$

with $\eta=\left\|\int_{-h}^{0} e^{A s} \mathrm{~d} s\right\|$. Similarly,

$$
\begin{aligned}
\hat{X}_{\hat{p}}(t)-x(t+h)= & e^{A h}[x(t)-e(t)]+\int_{t-h}^{t} e^{A(t-s)} B u(s) \mathrm{d} s \\
& +x(t)-e(t)-e^{A h}[x(t-h)-e(t-h)] \\
& -\int_{t-2 h}^{t-h} e^{A(t-h-s)} B u(s) \mathrm{d} s-x(t+h)
\end{aligned}
$$

Substituting $x(t+h)$ and $x(t)$ by their expressions from (24) leads to

$$
\begin{gathered}
\left.\hat{X}_{\hat{p}}(t)-x(t+h)=-e^{A h} e(t)-e(t)+e^{A h} e(t-h)\right] \\
-\int_{t-h}^{t} e^{A(t-s)}[d(s+h)-d(s)] \mathrm{d} s .
\end{gathered}
$$

Thus, the following maximization is derived

$$
\begin{gathered}
\|x(t)\| \leq\left\|\hat{X}_{\hat{p}}(t-h)\right\|+\eta h D_{\max }+\|e(t-h)\|+ \\
\left\|e^{A h}\right\|\|e(t-h)-e(t-2 h)\| .
\end{gathered}
$$


As a result, if (1) is controlled by the feedback $u(t)=f\left(\hat{x}_{\hat{p}}\right)$, the inequality

$$
\|x(t)\| \leq \beta e^{\alpha h} e^{-\alpha(t-T)}+\underbrace{\left[\eta+\Gamma\left\|e^{A h}\right\|[1+\gamma\|L C\|]\right] d_{\max }}_{r_{1}}
$$

holds and, if (1) is controlled by the feedback $u(t)=f\left(\hat{X}_{\hat{p}}\right)$, the inequality

$$
\begin{gathered}
\|x(t)\| \leq \beta e^{\alpha h} e^{-\alpha(t-T)} \\
+\underbrace{\Gamma[1+\gamma\|L C\|] d_{\max }+\left[\eta+\Gamma\left\|e^{A h}\right\|[1+\gamma\|L C\|]\right] h D_{\max }}_{r_{2}}
\end{gathered}
$$

is verified. This proves the following theorem.

Theorem 1: Consider system (1), observer (8) and predictor-controllers (13)-(16) and (12)-(20), resulting in error bounds respectively $r_{1}$ in (26) and $r_{2}$ in (27). If $D_{\max }>0$ and the bounds on $d(t)$ comply with the relations

$$
\frac{d_{\max }}{D_{\max }}>h \frac{\left\|e^{A h}\right\|}{\left\|e^{A h}\right\|-1},
$$

then one gets $r_{2}<r_{1}$ for $\left\|e^{A h}\right\|>1$.

In addition, $d_{\max }$ and $D_{\max }$ are supposed to be known in order to apply this criterion but they are not used in the controller design. Theorem 1 and equations (26) and (27) show that the design of a Lipschitz controller with the new reconstructed prediction $\hat{X}_{\hat{p}}$ given by (20) leads to a smaller error bound than designing a controller with the standard reconstructed prediction (16). Consequently, the new predictive scheme is said to better attenuate the disturbances than the standard one. Note that the criterion (28) is the same as in [1]: it is independent of the observer choice. Indeed, the same observer is designed in both cases: it means that whatever the observer (robust, finite time), (28) will remain unchanged. The result holds for a wide class of functions $f$ so it gives the possibility to robustly stabilize $\hat{X}_{\hat{p}}$ at zero. However, even if $\hat{X}_{\hat{p}}$ converges to zero, there are inevitable errors in (25) when the perturbation is timevarying:

- $\|e(t-h)\|+\left\|e^{A h}\right\|\|e(t-h)-e(t-2 h)\|$ is due to the observation error and it can be attenuated by the design of a robust observer;

- $\eta h D_{\max }$ is independent of the controller and the observer and cannot be reduced.

Note that if the observation error in (26) and (27) is canceled, we get back to the full state knowledge case like in [1]. Theorem 1 holds for time-varying disturbances, i.e when $D_{\max }>0$. Nevertheless, for constant disturbances, $r_{1}$ is not modified and $r_{2}$ becomes

$$
r_{2}=\Gamma[1+\gamma \| L C||] d_{\max } \quad \text { if } D_{\max }=0 ;
$$

so one always has $r_{2}<r_{1}$. These results are going to be illustrated in the next section.

\section{Simulation}

A. Model presentation, observer and predictor-controller design

A second order perturbed system has been chosen to illustrate the results. Its state space representation is

$$
\left\{\begin{array}{l}
\dot{x}(t)=\left[\begin{array}{cc}
0 & 1 \\
-a_{0} & -a_{1}
\end{array}\right] x(t)+\left[\begin{array}{l}
0 \\
1
\end{array}\right] u(t-h)+\left[\begin{array}{c}
0 \\
d(t)
\end{array}\right] \\
y(t)=x_{1}(t)
\end{array}\right.
$$

with $a_{0}=9$ and $a_{1}=-3$. The parameters chosen for all the simulations are $h=0.5 \mathrm{~s}, x(0)=[1.5,1]^{T}$ and $\hat{x}(0)=$ $[0,0]^{T}$. Two Luenberger observers are tested:

$$
\dot{\hat{x}}(t)=A \hat{x}(t)+B u(t-h)+L_{1}(y(t)-C \hat{x}(t))
$$

and

$$
\dot{\hat{x}}(t)=A \hat{x}(t)+B u(t-h)+L_{2}(y(t)-C \hat{x}(t))
$$

with $L_{1}$ and $L_{2}$ such that (31) is faster than (30). In the sequel, the components of a vector are denoted by the subscript "i". For instance, one has $x_{\hat{p}}=\left[x_{\hat{p} 1}, x_{\hat{p} 2}\right]^{T}$. The PID controllers used in the simulation are defined in Table I and the function $f$ is defined below

$$
f(\chi)=k_{p} \chi_{1}(t)+k_{d} \chi_{2}(t)+k_{i} \int_{0}^{t} \chi_{1}(s) d s .
$$

Controllers (33) and (34) are designed using the whole state in order to have a comparison point with observer-based controllers (35) and (36). Finally, two kinds of disturbances, $d_{1}$ and $d_{2}$, are chosen as shown on Figure 1. The analytic
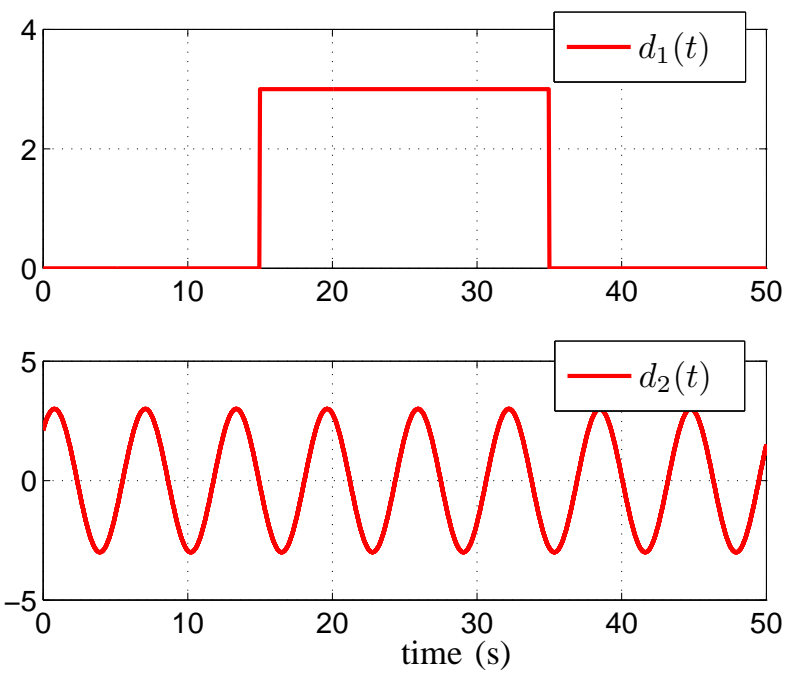

Fig. 1. Disturbances: $d(t)=d_{1}(t)$ or $d(t)=d_{2}(t)$

expression of $d_{2}$ is

$$
d_{2}(t)=3 \sin \left(t+\frac{\pi}{4}\right)
$$

so $d_{\max }=3$ and $D_{\max }=3$. 


\begin{tabular}{|c|c|c|}
\hline & STANDARD PREDICTIVE SCHEME & NEW PREDICTIVE SCHEME \\
\hline Full State Measurement & $u(t)=f\left(x_{\hat{p}}\right) \quad(33)$ & $u(t)=f\left(X_{\hat{p}}\right) \quad$ (34) \\
\hline Partial State Measurement & $u(t)=f\left(\hat{x}_{\hat{p}}\right) \quad$ (35) & $u(t)=f\left(\hat{X}_{\hat{p}}\right) \quad$ (36) \\
\hline
\end{tabular}

TABLE I

PRedictive FeEdbacks With $f$ Defined IN (32), $x_{\hat{p}}$ IN (5), $X_{\hat{p}}$ IN (4), $\hat{x}_{\hat{p}}$ IN (13), $\hat{X}_{\hat{p}}$ IN (12)

\section{B. Comparison of the schemes for constant disturbance $d_{1}$}

In this subsection, the performances of the closed-loop system (29) with one of the four controllers defined above are compared. From the analysis of the results obtained with "slow" observer (30) (see Figure 2), one concludes that:

- The controllers designed from the new predictions $X_{\hat{p}}$ and $\hat{X}_{\hat{p}}$ perform a better disturbance attenuation than the controllers from the standard ones: the convergence radius for solid lines is much smaller than for dotted ones.

- The controllers obtained from the reconstructed predictions $\hat{x}_{\hat{p}}$ (respectively $\hat{X}_{\hat{p}}$ ) do not achieve as good attenuation as the controllers from full state predictions $x_{\hat{p}}\left(\right.$ respectively $X_{\hat{p}}$ ): the convergence radius for black solid line (respectively dotted line) is larger than blue solid line (respectively dotted line).

- The observer-based controller (36) designed with the new reconstructed predictive scheme performs better disturbance attenuation than full state controller (33) designed with the standard predictive scheme. This confirms the improvement of the new predictive scheme to attenuate perturbation.

Note that in the full state knowledge case, the new predictive scheme (controller (34)) leads to the perfect rejection of the constant disturbance. In the case of partial measurement, this is not possible anymore because of the observation error. However, it is clear on Figure 3 (results obtained with "fast" observer (31)) that if the observer becomes faster the results from the reconstructed predictions tend to the full state knowledge case. Therefore, perfect rejection is almost achieved for the controller (36) from the new reconstructed scheme.

\section{Comparison of the schemes for time-varying disturbances $d_{2}$}

From the above subsection, it has been illustrated that the faster the observer is, the more attenuated is the perturbation. Therefore, only observer (31) has been used with the timevarying disturbance $d_{2}$. Note that criterion (28) is verified because $\frac{d_{\max }}{D_{\max }}=1$ and $h \frac{\left\|e^{A h}\right\|}{\left\|e^{A h}\right\|-1} \approx 0.58$. The only difference with perturbation $d_{1}$ is that, perfect rejection cannot be

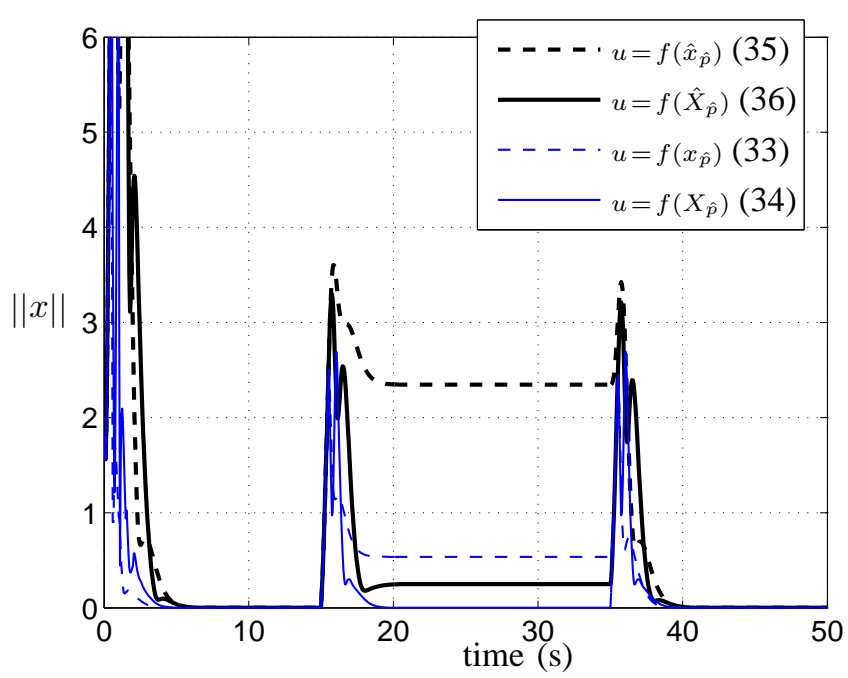

Fig. 2. Comparison between predictive feedbacks with "slow" observer (30) and disturbance $d_{1}$

achieved by a simple PID controller ${ }^{3}$ even if it is designed with the new predictive scheme and full state knowledge. Remark that it would not be possible in the delay free case either. The behaviour of the system with observer-based controllers (35) and (36) is diplayed on Figure 4. It is clear that the controller designed on the new predictive scheme performs better disturbance attenuation than the controller from the standard predictive scheme because the convergence radius for (36) is smaller than the convergence radius for (35). This observation confirms the result of Theorem 1.

\section{CONClusion}

It has been shown that the results of [1] can be extended to the case when only partial state measurement is available. In comparison with our previous work, an inevitable observation error is introduced but the new predictive scheme with the reconstructed state performs a better disturbance attenuation than the standard scheme with the reconstructed state. Besides, the faster the observer is, the closer the results

\footnotetext{
${ }^{3}$ It could have been possible to reject perfectly time-varying disturbances with sliding mode control for example but in this case $f$ is not Lipschitz anymore.
} 


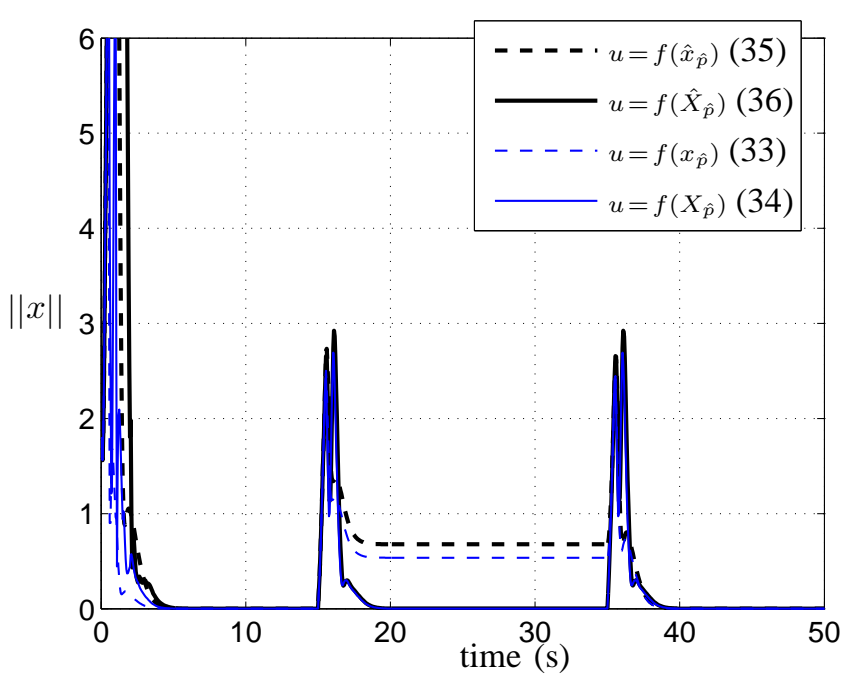

Fig. 3. Comparison between predictive feedback with "fast" observer (31) and disturbance $d_{1}$

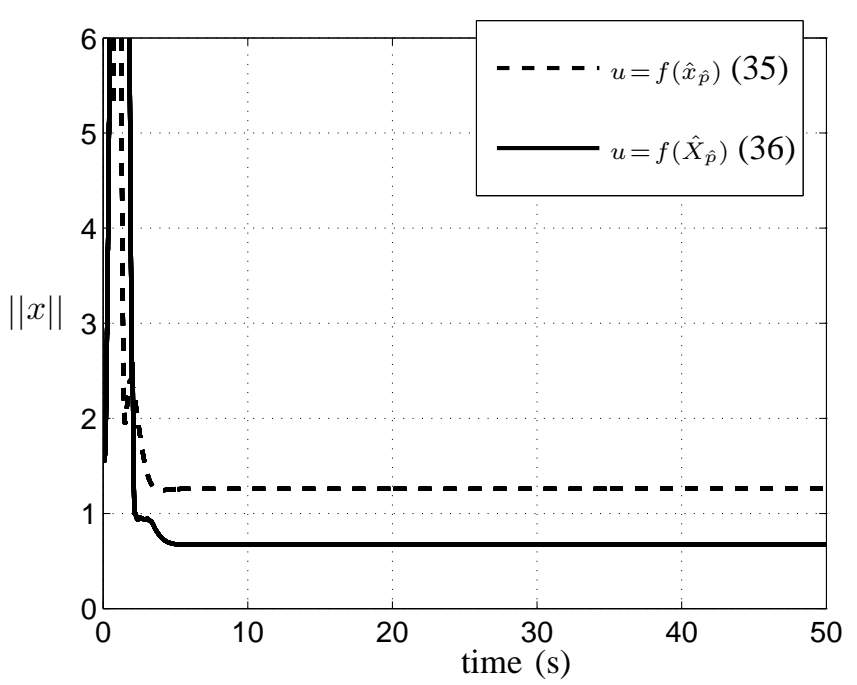

Fig. 4. Comparison between predictive feedback with "fast" observer (31) and disturbance $d_{2}$

get to the full state measurement case. This result is very interesting from a practical point of view. All the results are illustrated by simulations. An extension to unknown delay is one of the perspectives of this work.

\section{REFERENCES}

[1] V. Léchappé, E. Moulay, F. Plestan, A. Glumineau, and A. Chriette, "New predictive scheme for the control of LTI systems with input delay and unknown disturbances," To appear in Automatica. [Online]. Available: https://dl.dropboxusercontent.com/u/21853387/140352_Lechappe.pdf

[2] O. J. M. Smith, "Closer control of loops with dead time," Chemical Engineering Progress, vol. 53, no. 5, pp. 217-219, 1957.

[3] W. Kwon and A. Pearson, "Feedback stabilization of linear systems with delayed control," IEEE Transactions on Automatic Control, vol. 25, no. 2, pp. 266-269, 1980.

[4] A. Manitius and A. Olbrot, "Finite spectrum assignment problem for systems with delays," IEEE Transactions on Automatic Control, vol. 24, no. 4, pp. 541-552, 1979.
[5] Z. Artstein, "Linear systems with delayed controls: A reduction," IEEE Transactions on Automatic Control, vol. 27, no. 4, pp. 869-879, 1982.

[6] M. Krstic, Delay compensation for nonlinear, adaptive, and PDE systems, ser. Systems and Control: Fundations and Applications. Springer, 2009.

[7] A. Polyakov, "Minimization of disturbances effects in time delay predictor-based sliding mode control systems." J. Franklin Inst., vol. 349, no. 4, pp. 1380-1396, 2012.

[8] Y.-H. Roh and J.-H. Oh, "Sliding mode control with uncertainty adaptation for uncertain input-delay systems," International Journal of Control, vol. 73, no. 13, pp. 1255-1260, 2000.

[9] O. Camacho, R. Rojas, and W. Garcia-Gabin, "Some long time delay sliding mode control approaches," ISA Transactions, vol. 46, no. 1, pp. $95-101,2007$.

[10] X. Han, E. Fridman, and S. Spurgeon, "Sliding mode control in the presence of input delay: A singular perturbation approach," Automatica, vol. 48, no. 8, pp. 1904 - 1912, 2012.

[11] L. Fridman, E. Fridman, and E. Shustin, "Steady modes in relay systems with delay," in Sliding mode control in engineering, ser Automation and Control Engineering, W. Perruquetti and J.-P. Barbot, Eds. CRC Press, 2002.

[12] L. Mirkin and G. Tadmor, " $H_{\infty}$ control of system with i/o delay: a review of some problem oriented methods," IMA Journal of Mathematical Control and Information, vol. 19, no. 1 and 2, pp. 185-199, 2002.

[13] Q.-C. Zhong, Robust control of time-delay systems. Springer, 2006

[14] J. Klamka, "Observer for linear feedback control of systems with distributed delays in controls and outputs," Systems \& Control Letters, vol. 1, no. 5, pp. $326-331,1982$.

[15] K. Watanabe and M. Ito, "An observer for linear feedback control laws of multivariable systems with multiple delays in controls and outputs," Systems \& Control Letters, vol. 1, no. 1, pp. 54 - 59, 1981.

[16] D. Bresch-Pietri, J. Chauvin, and N. Petit, "Prediction-based feedback control of a class of processes with input-varying delay," in American Control Conference, Montréal, Canada, 2012.

[17] X. Zhang and Z. Cheng, "Output feedback stabilization of nonlinear systems with delays in the input," Applied Mathematics and Computation, vol. 167, no. 2, pp. 1026 - 1040, 2005.

[18] B. Zhou, Z.-Y. Li, and Z. Lin, "Observer-based output feedback control of linear systems with multiple input and output delays," in Conference on Decision and Control, Maui, USA, 2012, pp. 2370-2375.

[19] B. Zhou, Z. Lin, and G.-R. Duan, "Truncated predictor feedback for linear systems with long time-varying input delays," Automatica, vol. 48, no. 10, pp. 2387 - 2399, 2012.

[20] P. Albertos and P. Garcia, "Predictor-observer-based control of systems with multiple input/output delays," Journal of Process Control, vol. 22 , no. 7, pp. $1350-1357,2012$.

[21] M. D. Loreto, J.-J. Loiseau, and J.-F. Lafay, "Disturbance attenuation by dynamic output feedback for input-delay systems," Automatica, vol. 44, no. 8, pp. $2202-2206,2008$.

[22] V. Lakshmikanthan, S.Leela, and A.A.Martynyuk, Practical stability of nonlinear systems. Word scientific, 1990.

[23] H. Khalil, Nonlinear Systems. Prentice Hall PTR, 2002. 